\title{
Properties of ISM in two star-forming regions
}

\author{
Nikoghosyan E.*, Azatyan N., Harutyunian H., Baghdasaryan D., and Andreasyan D. \\ Byurakan Astrophysical Observatory, 0213, Aragatsotn prov., Armenia
}

\begin{abstract}
The Hi-GAL provides an opportunity to make a complete and unbiased view of the continuum emission in the Galactic plane in five bands: 70, 160, 250,350, and $500 \mu \mathrm{m}$. Our research focuses on two of star-forming regions. The first one is the molecular cloud, which includes G45.12+0.13 and G45.07+0.13 UC HII regions. Using the Modified blackbody fitting on Herschel images obtained in four bands: $160,250,350$, and $500 \mu \mathrm{m}$, we determined the distribution of $\mathrm{N}\left(\mathrm{H}_{2}\right)$ hydrogen column density and $\mathrm{T}_{d}$ dust temperature. The maps of $\mathrm{N}\left(\mathrm{H}_{2}\right)$ and $\mathrm{T}_{d}$ show that UC HII regions clearly stand out against the general background of the molecular cloud with a relatively low density (from $1.0 \times 10^{23}$ to $3.0 \times 10^{23} \mathrm{~cm}^{-2}$ ) and significantly higher temperature (up to $100 \mathrm{~K}$ ), what is fully consistent with the basic concept of UC HII regions about the presence of a hot, high mass stellar source and stellar wind, which leads to the blowing out of matter. The second one is the elongated star-forming region, which includes five stellar subgroups around IRAS $05184+3635$, $05177+3636,05168+3634,05162+3639$ and $05156+3643$ sources. Here, on the contrary, the $\mathrm{N}\left(\mathrm{H}_{2}\right)$ is noticeably higher (from $1.0 \times 10^{23}$ to $5.0 \times 10^{23} \mathrm{~cm}^{-2}$ ) than in the surrounding molecular cloud and the $\mathrm{T}_{d}$ does not exceed $25 \mathrm{~K}$.
\end{abstract}

Keywords: stars: pre-main sequence - infrared: stars - radiative transfer - ISM: hydrogen column density, dust temperature

\section{Introuction}

The Hi-GAL provides an opportunity to make a complete and unbiased view of the continuum emission in the Galactic plane in five bands: 70, 160, 250,350, and $500 \mu \mathrm{m}$. This range of wavelengths covers the peak of the spectral energy distribution (SED) of the cold dust emission and makes it possible to determine such important parameters of Interstellar medium (ISM) as the $\left.\mathrm{N}_{(} \mathrm{H}_{2}\right)$ hydrogen column density and $\mathrm{T}_{d}$ dust temperature (Molinari et al., 2016).

For our study we choose two, relatively distant regions of star formation, which are different according to their properties and stellar content. The first is a pair of UC HII regions G45.07+0.13 and G45.12+0.13, associated with IRAS $19110+1045$ and IRAS $19111+1048$ sources, respectively (Wood \& Churchwell, 1989). In Figure 1, we present the colour-composite image (left panel) with HiGAL three bands, where it is clearly seen that both regions are clearly distinguished by their brightness against the background of the surrounding molecular cloud. Moreover, it is clearly seen that they are connected by a relatively colder bridge. This can serve as further evidence that they belong to the same star-forming region and form a physically connected system. According to the results obtained in Han et al. (2015), the near and far kinematic distances of G45.07+0.13 and G45.12+0.13 regions are $\sim 4.0$ and $\sim 8.0 \mathrm{kpc}$ with tangent point distance of $\sim 6 \mathrm{kpc}$. The last, far distance estimation, is better consistent with a more recent estimate according to the trigonometric parallax $(7.75 \pm 0.45 \mathrm{kpc})$ in Wu et al. (2019). Following them, we adopt a distance of $7.8 \mathrm{kpc}$ in our study.

The next star-forming region is a molecular cloud surrounding the five IRAS sources: $05168+3634$, 05184+3635, 05177+3636, 05162+3639, and IRAS 05156+3643 (Azatyan, 2019). The right panel of Figure 1 presents the colour-composite image with Hi-GAL three bands, where we can see that there are relatively hot gas-dust matter concentrations in the vicinity of all IRAS sources. The distance

*elena@bao.sci.am, Corresponding author 

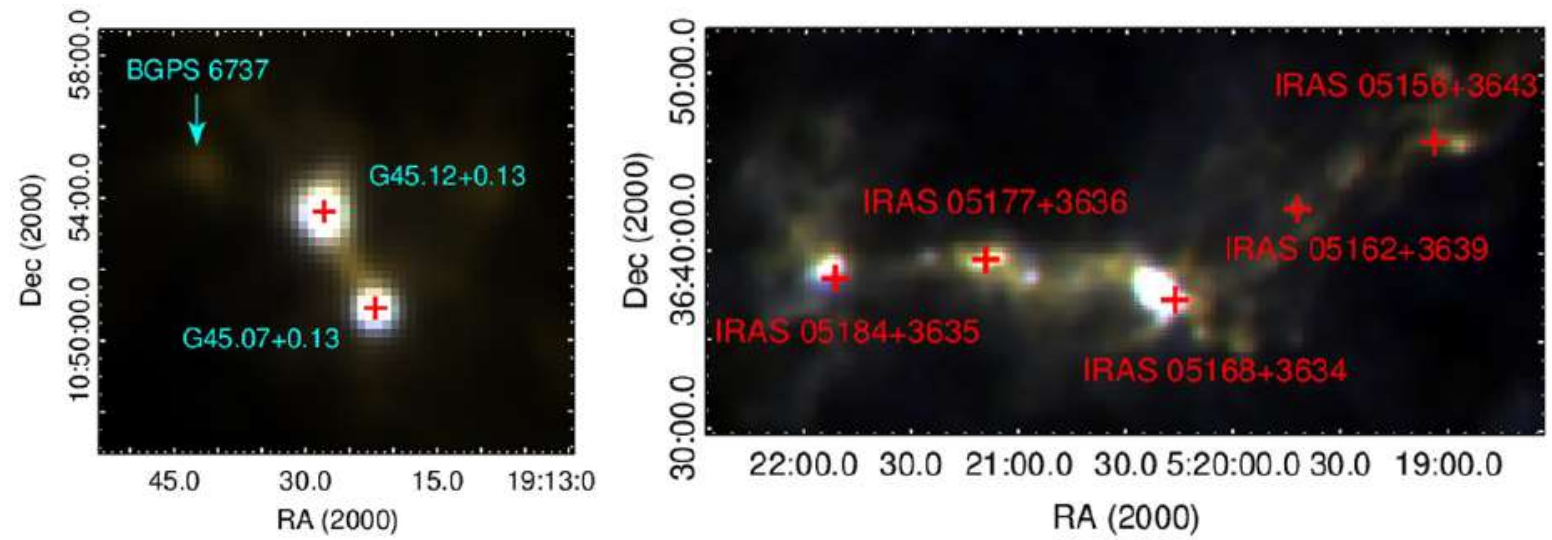

Figure 1. Colour-composite images with Hi-GAL three bands: PACS $160 \mu \mathrm{m}$ (blue), SPIRE $350 \mu \mathrm{m}$ (green), and $500 \mu \mathrm{m}$ (red). Left panel: G45.07+0.13 and G45.12+0.13 UC HII regions; right panel: IRAS $05168+3634$ (Mol 9) star-forming region. The positions of IRAS sources are marked by crosses.

estimations of this region are controversial. A kinematic distance estimated by Molinari et al. (1996) is $6.08 \mathrm{kpc}$. The distance of IRAS $05168+3634$ estimated according to a trigonometric parallax with VERA is $1.9 \pm 0.2 \mathrm{kpc}$ (Sakai et al., 2012). Such a large difference in the calculated distances determines a significant difference in the assessment of the mass of gas-dust matter. Therefore, for our calculations, we used both distance estimates.

The both star-forming regions are associated with different manifestations of activity, including maser and continual molecular emission, outflows, etc (Varricatt et al., 2010, and ref. therein). Our previous studies shown that all IRAS sources in both star-forming regions are associated with dense young stellar clusters. The dense stellar clusters are associated with both G45.07+0.13 and G45.12+0.13 UC HII regions include a number of high-mass ZAMS stellar objects (Azatyan et al., 2020, Rivera-Ingraham et al., 2010, Vig et al., 2006). The molecular cloud in the second star-forming regions includes 240 candidates of YSOs within the radii of subclusters around all five IRAS sources. The age of the subclusters is estimated at 0.1-3 Myr (Azatyan, 2019).

\section{Method}

To obtain the physical parameters like $\mathrm{N}\left(\mathrm{H}_{2}\right)$ and $\mathrm{T}_{d}$, the Modified single-temperature blackbody fitting, as well as the thermal emission from cold dust lying in the Herschel FIR optically thin bands $(160-500 \mu \mathrm{m})$ were used (Hildebrand, 1983). Following the discussion in the previous studies (e.g. Battersby et al., 2011), we excluded the $70 \mu \mathrm{m}$ observational data as the optically thin assumption would not hold. Besides, the emission here would have a significant contribution from the warm dust component, thus modelling with a single-temperature blackbody would over-estimate the derived temperatures. For initial Herschel images processing, which includes the elimination of bad pixels effect, the transformation of surface brightness units to Jy pixel ${ }^{-1}$, as well as the convolution of images resolution to the $500 \mu \mathrm{m}$ image (the lowest among all images), we used the HIPE software.

The Modified single-temperature blackbody fitting, which was subsequently carried out on a pixelby-pixel basis using the following formula:

$$
S_{\nu}=B_{\nu}\left(\nu, T_{d}\right) \Omega\left(1-e^{-\tau(\nu)}\right),
$$

with

$$
\tau(\nu)=\mu_{H_{2}} m_{H} k_{\nu} N\left(H_{2}\right),
$$

where $\nu$ is the frequency, $S_{\nu}(\nu)$ is the observed flux density, $B_{\nu}\left(\nu, T_{d}\right)$ is the Planck function, $\Omega$ is the solid angle in steradians from where the flux is obtained (in this case for all bands the solid angle subtended by a $14 \operatorname{arcsec} \times 14 \operatorname{arcsec}$ pixel), $\tau(\nu)$ is the optical depth, $\mu_{H_{2}}$ is the mean molecular weight (adopted as 2.8 here), $m_{H}$ is the mass of hydrogen, and $k_{\nu}$ is the dust opacity. 

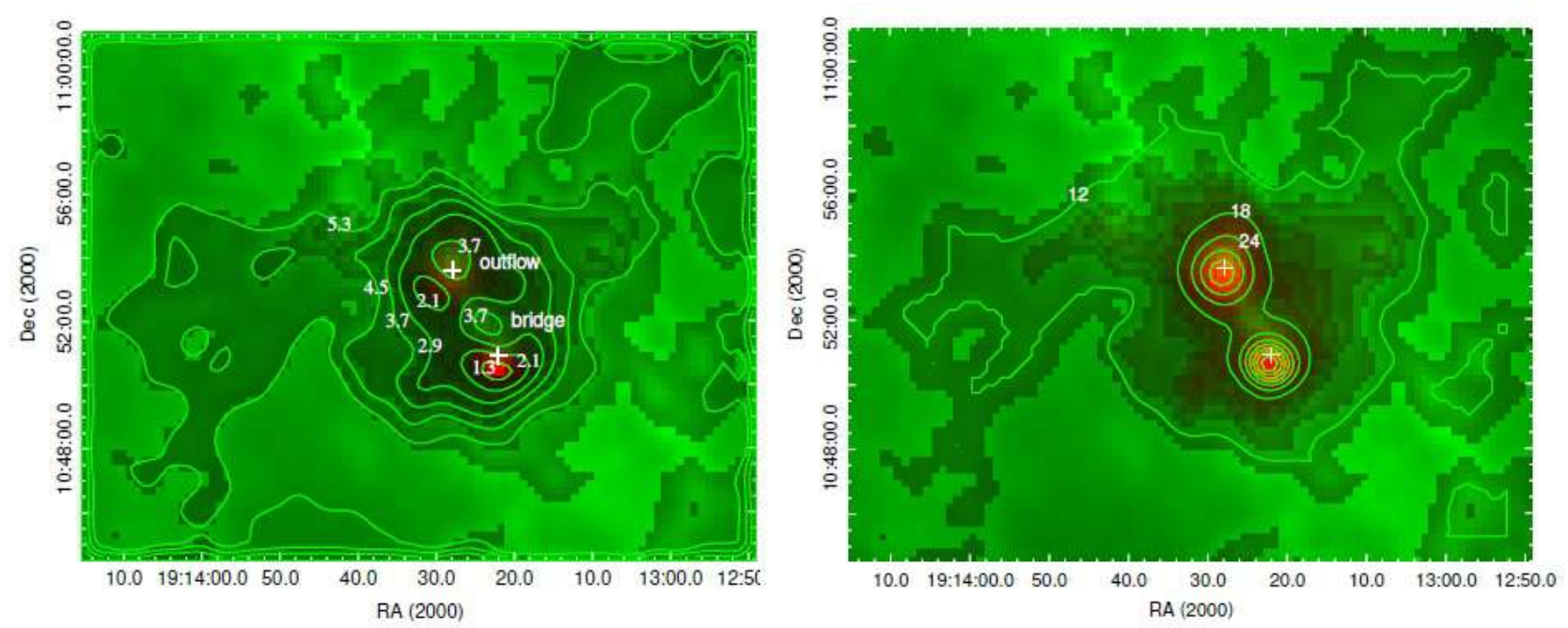

Figure 2. Colour-composite images of $\mathrm{T}_{d}$ (red) and $\mathrm{N}\left(\mathrm{H}_{2}\right)$ (green) distribution of the region surrounding G45.12+0.13 and G45.07+0.13 UC HII with overlaid contours of temperature in the left panel and $\mathrm{N}\left(\mathrm{H}_{2}\right)$ on the right panel. The temperature contours cover a range from 12 to $66 \mathrm{~K}$ with step in increments of $6 \mathrm{~K}$. The $\mathrm{N}\left(\mathrm{H}_{2}\right)$ contours cover a range from $1.3 \times 10^{23}$ to $5.3 \times 10^{23} \mathrm{~cm}^{-2}$ with step in increments of $0.8 \times 10^{23} \mathrm{~cm}^{-2}$. The positions of IRAS sources are marked by white crosses. On the right panel, the positions of G45.12+0.13 outflow and bridge between G45.12+0.13 and G45.07.0.13 UC HII are indicated (for more detail information see text).

For opacity, we adopted a functional form of $k_{\nu}=0.1(\nu / 1000 \mathrm{GHz})^{\beta} \mathrm{cm}^{2} \mathrm{~g}^{-1}$, with $\beta=2$ (see Hildebrand, 1983). For each pixel, equation (1) was fitted using the four data points $(160,250,350$, and $500 \mu \mathrm{m}$ ) keeping $\mathrm{T}_{d}$ and $\mathrm{N}\left(\mathrm{H}_{2}\right)$ as free parameters. Launhardt et al. (2013) used a conservative $15 \%$ uncertainty in the flux densities of the Herschel bands. We adopted the same value here for all bands. The uncertainties of the parameters were derived using Pearson's $\chi^{2}$ statistics:

$$
\chi^{2}=\sum_{i=1}^{N} \frac{\left(D_{i}-F_{i}\right)^{2}}{F_{i}},
$$

where $\mathrm{D}_{i}$ is the observed flux and $\mathrm{F}_{i}$ is the flux predicted by the model, $\mathrm{N}$ is the number of bands.

From the derived column density values, we estimate the mass of the dusty clumps using the following expression:

$$
M_{\text {clump }}=\mu_{H_{2}} m_{H} \text { Area }_{\text {pix }} \sum N\left(H_{2}\right)
$$

where Area $_{\text {pix }}$ is the area of a pixel in $\mathrm{cm}^{2}$.

\section{Results}

The results for both star-forming regions (see Sec. 1) are presented below.

\subsection{G45.07+0.13 and G45.12+0.13 UC HII regions}

The final obtained column density and dust temperature maps of the wider region surrounding G45.12+0.13 and G45.07+0.13 UC HII objects are shown in Figure 2. The map of temperature distribution clearly shows that the centers of both UC HII regions practically coincide with the temperature maxima, which reaches $49 \mathrm{~K}$ in $\mathrm{G} 45.12+0.13$ and $97 \mathrm{~K}$ in $\mathrm{G} 45.07+0.13$. On average, in the vicinity of IRAS $19110+1045$, the value of $\mathrm{N}\left(\mathrm{H}_{2}\right)$ is $\sim 2 \times 10^{23} \mathrm{~cm}^{-2}$, which is well coincides with the data in Churchwell et al. (2010) and almost an order of magnitude less than it was estimated from the $1.3 \mathrm{~mm}$ dust emission in Hernández-Hernández et al. (2014). On the other hand, the lower temperature estimation in the same work $(82 \mathrm{~K})$ is well consistent with ours. 

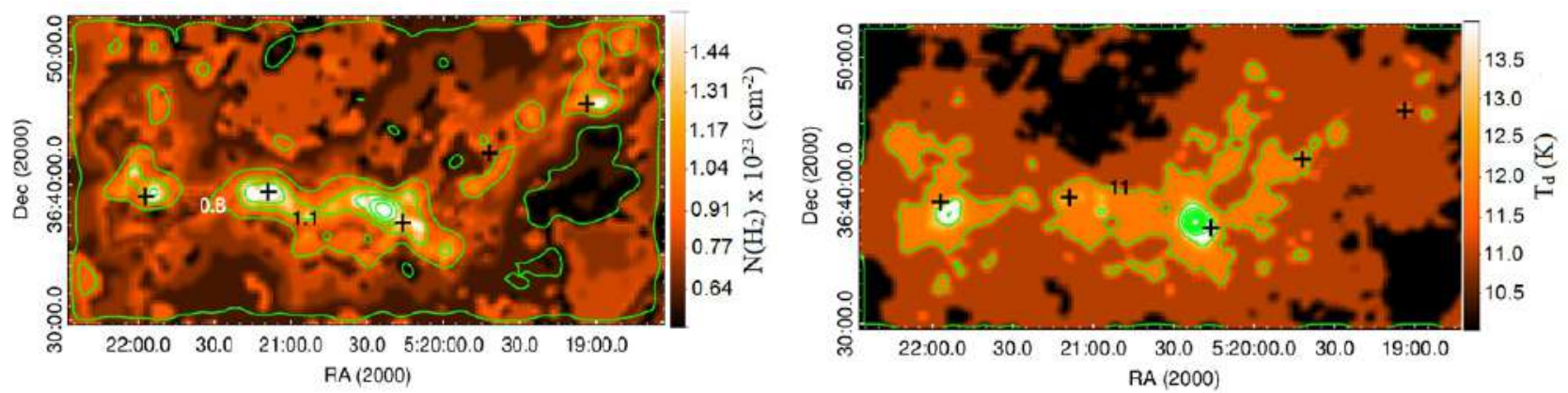

Figure 3. Maps of column density (left panel) and dust temperature (right panel) of IRAS $05168+3634$ star-forming regions. On the $\mathrm{N}\left(\mathrm{H}_{2}\right)$ map the isodenses corresponding to the values of $1.1 \times 10^{23}$ and $0.8 \times 10^{23} \mathrm{~cm}^{-2}$ shown. On $\mathrm{T}_{d}$ map isotherm corresponding to the value of $11 \mathrm{~K}$ is shown. The positions of IRAS sources are marked by crosses.

In the vicinity of IRAS $19111+1048, \mathrm{~N}\left(\mathrm{H}_{2}\right)$ is equal to $\sim 4 \times 10^{23} \mathrm{~cm}^{-2}$ that well consistents with the data of multi transition ${ }^{13} \mathrm{CO}$ and $\mathrm{CS}$ observations in the G45.12+0.13 core (Churchwell et al., 1992). IRAS $19111+1048$ itself is located in a region with a relatively high column density, which coincides in coordinates with counters of blueshifted emission of the bipolar molecular outflow (Hunter et al., 1997). It can be assumed that the outflow is the cause of the formation of the region with increased $\mathrm{N}\left(\mathrm{H}_{2}\right)$. It should be noted one more fact. One more region with a relatively high density and low temperature is located between the two UC HII regions. This region by coordinates coincides with the bridge between the UCHII regions, which is clearly distinguishable on Herschel colourcomposite image (see Figures 1). This bridge is also clearly visible on the H30 $\alpha$ map in Churchwell et al. (2010).

As can be seen in Figure 2, the temperature distribution in both UC HII regions has a pronounced spherical symmetry, and at a distance of 7 pix $(\sim 1.6 \operatorname{arcmin}$ or $\sim 3.7 \mathrm{pc})$ in G45.12+0.13 and 5 pix $(\sim 1.2$ arcmin or $2.6 \mathrm{pc})$ in G45.07+0.13 from IRAS sources, the temperature decline almost stops. These distances correspond to $18 \mathrm{~K}$ isotherm and $2.9 \times 10^{23} \mathrm{~cm}^{-2}$ isodense, after which the column density begins to increase rapidly. We can assume that it is the region where the influence of the stellar wind, leading to blowing of matter, ends. The total masses of these regions were calculated by Formula 4 for G45.12+0.13 region is $\sim 2.2 \times 10^{5} \mathrm{M}_{\odot}$ and for $\mathrm{G} 45.07+0.13$ is $10^{5} \mathrm{M}_{\odot}$.

It should be noted that the parameters obtained by the Modified blackbody model are not without certain errors (Harutyunyan et al., 2020). Among other causes the errors are a strong function of the parameters' values. In general, the $\chi^{2}$ in the UCHII regions does not exceed 0.1. However, the uncertainty of parameters significantly increases in the regions with the maximum temperature, reaching a value of 0.8 .

Table 1. The parameter of gas-dust matter and stellar content in the vicinity of IRAS sources

\begin{tabular}{lccccc}
\hline Parameter & $05156+3643$ & $05162+3639$ & $05168+3634$ & $05177+3636$ & $05184+3635$ \\
\hline Radius (arcmin) & 2.8 & 0.25 & 3.0 & 3.5 & 2.5 \\
YSO's number & 47 & 5 & 57 & 79 & 52 \\
$\mathrm{~N}\left(\mathrm{H}_{2}\right) \times 10^{23} \mathrm{~cm}^{-2}$ & 1.2 & 0.8 & 1.8 & 1.7 & 1.5 \\
$\mathrm{~T}_{d}(\mathrm{~K})$ & 12 & 12 & 24 & 13 & 14 \\
$\mathrm{M}\left(\mathrm{M}_{\odot}\right)$ for d $=1.9 \mathrm{kpc}$ & $1.2 \times 10^{4}$ & $1.9 \times 10^{2}$ & $1.9 \times 10^{3}$ & $2.2 \times 10^{4}$ & $1.0 \times 10^{4}$ \\
$\mathrm{M}\left(\mathrm{M}_{\odot}\right)$ for d $=6.1 \mathrm{kpc}$ & $1.4 \times 10^{5}$ & $2.2 \times 10^{3}$ & $2.1 \times 10^{4}$ & $2.5 \times 10^{5}$ & $1.2 \times 10^{5}$ \\
\hline
\end{tabular}




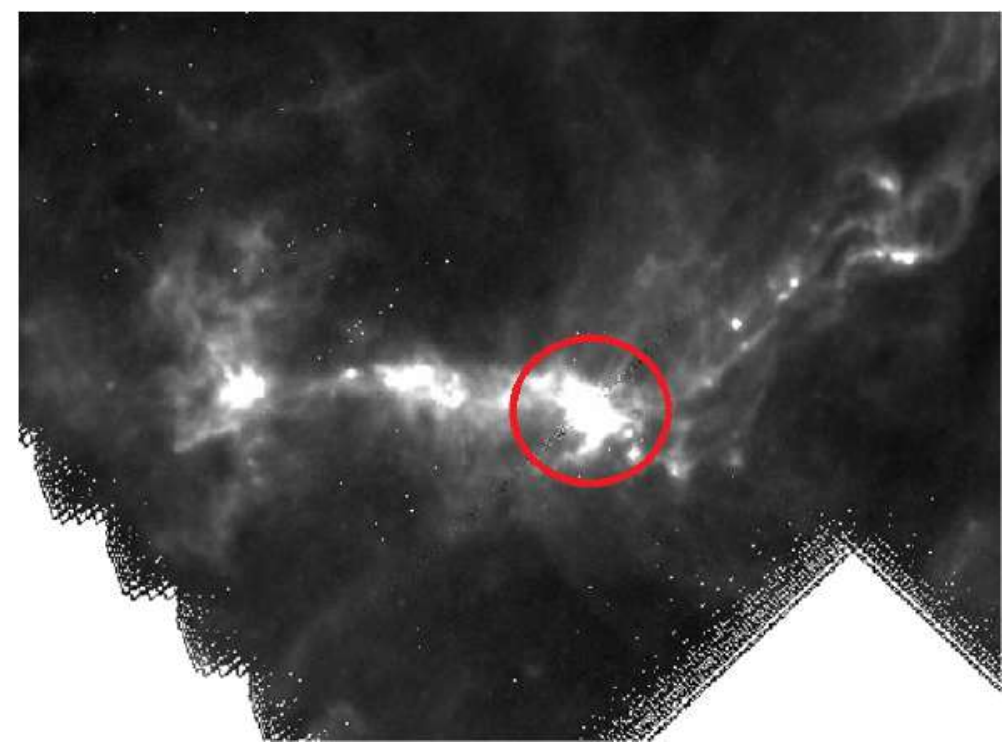

Figure 4. 2.5 level $250 \mu \mathrm{m}$ Herschel image of IRAS $05168+3634$ star-forming region. The red circle marks the vicinity of IRAS $05168+3634$ source.

\subsection{IRAS $05168+3634$ star-forming region}

The final obtained column density and dust temperature maps of the wider region surrounding five IRAS sources $(05168+3634,05184+3635,05177+3636,05162+3639$, and IRAS $05156+3643)$ are shown in Figure 3. In contrast to the previous region, this star-forming region stand out against of the environment not only with a higher temperature, but also with a higher density. In general, $\mathrm{N}\left(\mathrm{H}_{2}\right)$ in molecular clouds surrounding IRAS sources changes from $\sim 0.8 \times 10^{23}$ to $\sim 2.7 \times 10^{23} \mathrm{~cm}^{-2}$, and $\mathrm{T}_{d^{-}}$ from 11 to $26 \mathrm{~K}$. We can see that the density in this star-forming region is only slightly less than in the previous one. However, the temperature of the gas-dust matter is much lower. Such a significant difference, of course, is primarily due to the stellar content. As mentioned above, stellar clusters in the UCHII regions include a significant number of high mass ZAMS stellar objects, accordingly with higher temperatures. In contrast, the subclusters in IRAS 05168+3634 star-forming region are mainly composed of younger, middle- and low-mass stellar objects with accordingly lower temperatures (Azatyan, 2019). The Table 1 lists some parameters that characterize both the stellar population and the gas-dust matter in the IRAS subclusters: the radii of the subclusters and the number of YSOs identified in them, as well as the hydrogen column density, dust temperature, and mass of the gas-dust component. Based on these data, a relationship is observed between the number of identified YSOs and the mass of the gas-dust component. Undoubtedly, the relationship between these parameters, including the evolutionary stage of the stellar objects, requires a more detailed study.

In general, in the molecular cloud, the uncertainty of parameters does not exceed 0.02. In general, the value of $\chi^{2}$ is in this star-forming region noticeably lower than in the UC HII regions. This is most likely due to the fact that the temperature regime in this region is more suitable for the model we have applied. However, in the central part, in the vicinity IRAS $05168+3634$, the uncertainty increases significantly, up to 0.9 . In this case, this cannot be explained by the high temperature. The increase in the uncertainly of the parameters in this case is most likely explained by the quality of the images. Unfortunately, in all available Herschel images, this star-forming region is located at the very edge, where, of course, the image quality, especially in the $250 \mu \mathrm{m}$ channel, is not good enough. The Figure 4 clearly shows that in the area around the IRAS $05168+3634$ source, even in a 2.5 level image, there are many bad pixels that, most likely, is the reason for the increase in the $\chi^{2}$ value.

\section{Conclusion}

Thus, the study of ISM in both star-forming regions of showed that with a small difference in density, they differ significantly in temperature. Undoubtedly, this difference is interconnected with 
the stellar composition. This once again confirms the well-known fact that any problem in astronomy, including the process of star formation, should be studied by a combination of many factors on the basis of extensive observational data, which can only be provided by survey observations made in different spectral ranges.

\section{Acknowledgements}

The authors are grateful to the organizers for holding the International Symposium "Astronomical Surveys and Big Data 2" and for publishing the paper.

\section{References}

Azatyan N. M., 2019, A\&A, 622, A38

Azatyan N., Nikoghosyan E., Harutyunian H., Baghdasaryan D., Andreasyan D., 2020, ComBAO, 67, in press

Battersby C., et al., 2011, A\&A, 535, A128

Churchwell E., Walmsley C. M., Wood D. O. S., 1992, A\&A, 253, 541

Churchwell E., Sievers A., Thum C., 2010, A\&A, 513, A9

Han X. H., Zhou J. J., Wang J. Z., Esimbek J., Zhang J. S., Wang N., 2015, A\&A, 576, A131

Harutyunyan H. A., Nikoghosyan E. H., Azatyan N. M., 2020, Communications of the Byurakan Astrophysical Observatory, 67, 8

Hernández-Hernández V., Zapata L., Kurtz S., Garay G., 2014, ApJ, 786, 38

Hildebrand R. H., 1983, QJRAS, 24, 267

Hunter T. R., Phillips T. G., Menten K. M., 1997, ApJ, 478, 283

Launhardt R., et al., 2013, A\&A, 551, A98

Molinari S., Brand J., Cesaroni R., Palla F., 1996, A\&A, 308, 573

Molinari S., et al., 2016, A\&A, 591, A149

Rivera-Ingraham A., et al., 2010, ApJ, 723, 915

Sakai N., Honma M., Nakanishi H., Sakanoue H., Kurayama T., Shibata K. M., Shizugami M., 2012, PASJ, 64, 108

Varricatt W. P., Davis C. J., Ramsay S., Todd S. P., 2010, MNRAS, 404, 661

Vig S., Ghosh S. K., Kulkarni V. K., Ojha D. K., Verma R. P., 2006, ApJ, 637, 400

Wood D. O. S., Churchwell E., 1989, ApJS, 69, 831

Wu Y. W., et al., 2019, ApJ, 874, 94 